\section{Conocimiento especializado de los estudiantes para profesor de primaria en la resolución de problemas de proporcionalidad compuesta basada en las unidades de medida}

\author{
Prospective Primary teachers`specialised knowledge when \\ solving compound proportionality problems based on mea-
}

surement units

\section{BEATRIZ PÉREZ-BUENO \\ MARÍA DEL MAR LIÑÁN-GARCÍA \\ VÍCTOR J. BARRERA-CASTARNADO \\ Centro de Estudios Universitarios Cardenal Spínola CEU.}

\section{RESUMEN}

Considerando la importancia del razonamiento proporcional en la formación matemática, y teniendo en cuenta resultados de investigaciones que muestran las dificultades que genera el desarrollo del mismo, pretendemos identificar aspectos del conocimiento especializado movilizado en la resolución de un problema de proporcionalidad compuesta por cuatro estudiantes para profesor de Educación Primaria. Utilizando el modelo de Conocimiento Especializado del Profesor de Matemáticas (MTSK, Mathematics Teachers' Specialised Knowledge) analizamos los métodos de resolución usados desde las evidencias e indicios de dicho conocimiento así como desde las oportunidades que nos permiten identificar el conocimiento evocado por las respuestas. Los resultados reflejan que las resoluciones basadas en las unidades de medida de las magnitudes involucradas muestran un conocimiento más rico.

\section{ABSTRACT}

Considering the importance of proportional reasoning in the mathematical training, and taking into account papers that show the difficulties generated by the development of this knowledge, we try to identify the specialised knowledge movilised in the resolution of a compound proportion problem of four prospective primary teachers. Using the Mathematics Teachers' Specialised Knowledge model (MTSK) we analyse the resolution methods used, detecting evidences and traces of such knowledge, as well as the opportunities that allow us to identify the knowledge evoked by the answers. The results show a richer knowledge when using resolutions based on the magnitude measure units.

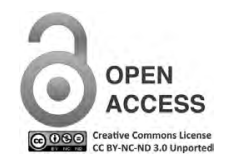

Para citar este artículo: Pérez-Bueno, B., Liñán-García, M. M. y BarreraCastarnado, V. J. (2018). Conocimiento especializado de los Estudiantes para Profesor en la resolución de problemas de proporcionalidad compuesta basada en las unidades de medida. EA, Escuela Abierta, 21, 47-64. doi: 10.29257/EA21.2018.04
Recibido: 17/07/2017

Aceptado: 08/02/2018

PALABRAS CLAVES

Conocimiento especializado del profesor de Matemáticas (MTSK), proporcionalidad compuesta, estudiantes para profesor, unidades de medida.

\section{KEYWORDS}

Mathematics Teachers' Specialised Knowledge (MTSK), compound proportion, prospective primary teachers, measurement units. 


\section{JUSTIFICACIÓN}

No se puede poner en duda la importancia que tiene el estudio de la proporcionalidad en el campo de la Educación Matemática. De hecho, el NCTM (2000) remarca que este conocimiento es el punto de encuentro de diversos temas en los currículos oficiales, no solo porque supone la culminación de la aritmética elemental sino, además, porque el razonamiento proporcional es necesario para la adquisición de otros conocimientos posteriores más complejos (Rivas, Godino y Castro, 2012), como la semejanza geométrica, las ecuaciones lineales, la probabilidad, el análisis de datos, etc. Sin embargo, diferentes investigaciones muestran las dificultades que encuentran los estudiantes de distintos niveles educativos, incluidos estudiantes para profesor ${ }^{1}$ (en adelante, EPP), al afrontar situaciones de proporcionalidad. Cabe destacar, tal y como apuntan Valverde y Castro (2009), los obstáculos que se encuentran los EPP al comprender la relación funcional entre magnitudes proporcionales y, por tanto, las limitaciones que tienen para argumentar sobre los procesos de resolución. Esto también conlleva la falta de competencias para interpretar de manera adecuada las respuestas de los alumnos de primaria a problemas de proporcionalidad (Rivas et. al, 2012; Buforn, Fenández y Llinares, 2015). Esto hace que la gran mayoría de los profesores sigan utilizando procedimientos rutinarios como la regla de tres y el producto en cruz (Buforn y Fenández, 2014), especificando exclusivamente el cómo pero no el cuándo, ni el porqué.

Por otro lado, la mayoría de estas investigaciones se han centrado en situaciones de proporcionalidad simple, por lo que vemos necesario ampliar el campo a aquellos contextos en los que tres o más medidas de magnitud tienen una relación de proporcionalidad, lo que llamaremos proporcionalidad compuesta (González y Gómez, 2011). En este sentido, algunas investigaciones muestran estrategias en la resolución de problemas de proporcionalidad compuesta (Martínez, Muñoz y Oller, 2015) o los contenidos al respecto en los libros de texto (Martínez, Muñoz, Oller y Ortega, 2017), si bien otras plantean propuestas didácticas para trabajar este contenido en formación inicial de profesores (Barrera-Castarnado, Liñán-García y Pérez-Bueno, 2017). Siguiendo estos dos últimos hilos, hemos planteado en nuestras aulas la resolución de problemas a partir de la reflexión sobre las magnitudes que intervienen en base a sus unidades de medida, poniendo especial énfasis en el conocimiento de múltiples procesos resolutivos (Star y Rittle-Johnson, 2009).

Consideramos, como Carrillo, Climent, Montes et al. (2018), que todo el conocimiento que posee un profesor de matemáticas es especializado por ser inherente a su profesión. Estos autores afirman, además, que la profesión de profesor se emprende en su formación inicial y, por tanto, comienza en su etapa universitaria. Como consecuencia, esta investigación seguirá el modelo del Conocimiento Especializado del Profesor de Matemáticas (MTSK) propuesto por los citados autores, con objeto de comprender el conocimiento que se moviliza cuando los EPP resuelven problemas de proporcionalidad compuesta. Pretendemos, además, a través del análisis, establecer las posibles interrelaciones que se evidencien entre elementos de dicho conocimiento. Esto, a su vez, nos dará la oportunidad de diseñar propuestas de formación consistentes con los resultados obtenidos en este estudio.

Nuestro objetivo en este estudio es, por tanto, identificar aspectos del conocimiento especializado puesto en juego por EPP en la resolución de problemas de proporcionalidad numérica compuesta al usar diferentes métodos de resolución, alguno de ellos basado en el uso explícito de unidades de medida de las magnitudes que intervienen en los enunciados. 


\section{MARCO TEÓRICO}

Partiendo de Schoenfeld (2010, p. 25), entendemos el conocimiento de un individuo como la información que tiene disponible para usar para resolver problemas, alcanzar metas, o desarrollar cualquier tarea. jNótese que, de acuerdo a esta definición, el conocimiento no ha de ser necesariamente correcto! Este conocimiento contempla su competencia matemática interrelacionando la comprensión conceptual, la fluidez de conocimientos y el razonamiento flexible (Schoenfeld y Kirpatrick, 2008), lo que se torna, como veremos más adelante, de especial interés en nuestro trabajo, pues implica que tal conocimiento incluye saber por qué se hace y diferentes formas de hacerlo (Star y Rittle-Johnson, 2009).

Entendiendo el conocimiento profesional del profesor como aquel que este necesita y utiliza (Schoenfeld, 2010) por la naturaleza de la enseñanza de las matemáticas, nos posicionamos con Carrillo, Climent, Montes et al. (2018) al entender que tal conocimiento es especializado y no es compartido con otras ciencias o profesiones. No se consideran, por tanto, aspectos propios de la Didáctica y Psicología. Elegimos, consecuentemente, el modelo del Conocimiento Especializado del Profesor de Matemáticas (figura 1), MTSK, que se ha ido estructurando en distintos trabajos de investigación, en diferentes niveles educativos (Aguilar, 2015; Escudero-Ávila, 2015; Flores-Medrano, 2015; Montes, 2015; Moriel-Junior, 2014; Muñoz-Catalán, Liñán-García y Ribeiro, 2017; Ribeiro, Muñoz-Catalán, Liñán-García, 2015; Rojas, 2014; Vasco, 2015), llegando a la definición que vamos a utilizar en el presente trabajo de investigación (Carrillo, Climent, Montes et al., 2018).

Este modelo se sustenta en Shulman (1986) y Ball, Thames y Phelps. (2008); como estos, mantiene la diferenciación entre el conocimiento matemático (Mathematical Knowledge, MK) y el conocimiento didáctico del contenido matemático (Pedagogical Content Knowledge), si bien propone una categorización diferente dentro de cada dominio. Nos centraremos en el primero, pues será el que dará sustento a esta investigación (figura 1).

\section{Figura 1}

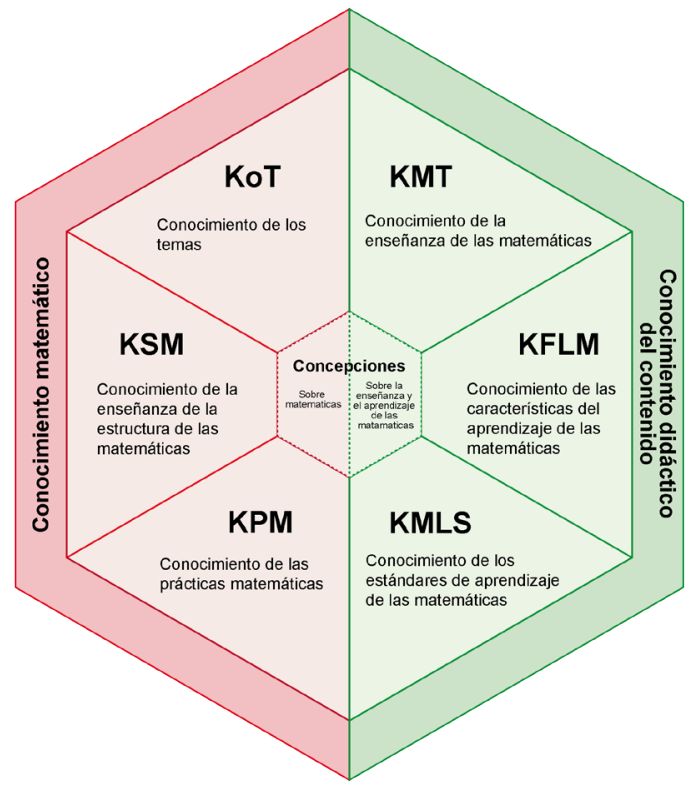


El conocimiento matemático (MK) contempla la propia disciplina matemática que se enseña; en nuestro caso, la proporcionalidad numérica. Está dividido en tres subdominos, siendo el primero de ellos el Conocimiento de los Temas Matemáticos (KoT). Partiendo del conocimiento profundo de la matemática fundamental (Ma, 1999), abarca el conocimiento de los contenidos, procesos y procedimientos matemáticos en sí mismos. Está separada en cuatro categorías: definiciones, propiedades y sus fundamentos; fenomenología; registros de representación y procedimientos.

Las Definiciones, Propiedades y sus Fundamentos consideran el conjunto de propiedades que hacen definible a un tema y le dan sentido y significado, además de las formas alternativas de hacerlo. Por ejemplo, podemos ubicar en esta categoría el conocimiento de las magnitudes entre las que se establece una relación de proporcionalidad, tanto directa como inversa, y las propiedades de las mismas, así como de las unidades de medida elegidas para medir cada una de ellas. Consideramos dentro de este subdominio las conexiones intraconceptuales, es decir, aquellas relaciones entre conceptos o procesos de un mismo tema, como por ejemplo, la relación entre las definiciones de razón de proporcionalidad funcional y escalar.

La fenomenología comprende los conocimientos del profesor sobre los fenómenos relacionados con un tema y los usos y aplicaciones del mismo. Por ejemplo, el uso de la razón de proporcionalidad funcional como factor de conversión entre unidades de medida.

Los registros de representación refieren el conocimiento del profesor sobre distintas representaciones: simbólica, gráfica, verbal, situaciones reales y manipulativa o concreta (Lesh, 1997), analítica, etc., y las transformaciones entre ellas sin pérdida de propiedades o características, lo que Star y Rittle-Johnson (2009) identifican como una de las interpretaciones de la flexibilidad matemática. Por ejemplo, representar la razón de proporcionalidad directa gráficamente o simbólicamente como la pendiente de una recta, o la equivalencia de la representación de la proporción por reducción a la unidad o razón escalar, ambas dentro del registro simbólico en este caso.

En la categoría procedimientos tenemos el conocimiento del cómo se hace, del cuándo, del porqué y de las características del resultado. Se recogerían en esta categoría tanto los algoritmos tradicionales como cualquier otro para un determinado contenido matemático. Por ejemplo, el conocimiento de qué es, cuándo se puede usar, cómo se calcula, por qué funciona, y qué significa el resultado obtenido en una regla de tres directa o inversa, dejando de ver esta como una mera mecanización de resolución de problemas.

El Conocimiento de la Estructura Matemática (KSM) incluye las conexiones interconceptuales del conocimiento del profesor sobre las relaciones entre distintos contenidos (Carrillo, Climent, Montes et al., 2018). Se distinguen cuatro categorías, empezando por las conexiones de complejización, que relacionan contenidos actuales con futuros. Podemos ver claros ejemplos en el profesor de Infantil o primer curso de Primaria cuando trabaja la medida desde unidades no estándar y establece partes proporcionales de la misma, como precursor de la medida en sí misma o la proporcionalidad directa como precursor de la función lineal. Las conexiones de simplificación relacionan contenidos actuales con otros anteriores. Por ejemplo, la conexión que permite fundamentar las relaciones de proporcionalidad entre magnitudes atendiendo a las que se establecen entre sus unidades de medida. Las conexiones transversales se observan entre contenidos diferentes que tienen cualidades en común. Por ejemplo, en el contexto de la semejanza de triángulos, existe una conexión transversal entre la proporción directa entre las medidas de sus lados y la igualdad de las amplitudes de los ángulos correspondientes, relacionando la proporcionalidad numérica con la proporcionalidad geométrica. Las conexiones auxiliares se establecen entre aquellos conocimientos que se relacionan entre sí como apoyo. Por ejemplo, el uso de la razón de proporcionalidad como herramienta para el cálculo de la probabilidad a través de la Regla de Laplace. 
El Conocimiento de las Prácticas Matemáticas (KPM), referidas a las acciones puestas en juego cuando se hacen matemáticas, como la resolución de problemas, la definición o demostración de un proceso o el uso correcto del lenguaje y los símbolos, etc. Actualmente este subdominio solo está caracterizado en función de indicadores (Carrillo, Climent, Montes, et al., 2018): jerarquización y planificación como forma de proceder en la resolución de problemas matemáticos, formas de validación y demostración, papel de los símbolos y uso del lenguaje formal, procesos asociados a la resolución de problemas como forma de producir matemáticas, prácticas particulares del quehacer matemático, $y$, finalmente, condiciones necesarias y suficientes para generar definiciones.

El segundo dominio del MTSK, conocimiento didáctico del contenido (Pedagogical Content Knowledge, PCK), caracteriza el conocimiento propio de la enseñanza. Puesto que no será utilizado en este trabajo, solo mencionaremos que está dividido en tres subdominios: el Conocimiento de las Características del aprendizaje de las matemáticas (Knowledge of Features of Learning Mathematics, KFLM), el Conocimiento de la Enseñanza de las Matemáticas (Knowledge of Mathematics Teaching, KMT) y el Conocimiento de los Estándares de Aprendizaje de las Matemáticas (Knowledge of Mathematics Learning Standards, KMLS).

\section{METODOLOGÍA}

Resultados derivados de investigaciones sobre resolución de problemas en los que intervienen relaciones de proporcionalidad numérica (Martínez et al., 2017; Sánchez, 2013; Fernández y Llinares, 2011; Valverde y Castro, 2009), así como de nuestra experiencia como formadores de maestros, han provocado que planteemos en nuestras aulas la resolución de este tipo de problemas tomando como base las relaciones entre las unidades de medida de las cantidades de magnitud que intervienen en el enunciado. Así, pretendemos evitar la mecanización de procedimientos sin comprensión de las relaciones establecidas entre magnitudes directa o inversamente proporcionales.

Como se avanzó en la introducción, nuestro objetivo principal es identificar aspectos del conocimiento especializado puesto en juego por EPP en la resolución de problemas de proporcionalidad numérica compuesta al usar diferentes métodos de resolución. Nos interesa, por tanto, dar respuesta a la siguiente pregunta de investigación ¿qué conocimiento especializado puede identificarse en el análisis de producciones de EPP cuando resuelven problemas de proporcionalidad numérica compuesta? El modelo analítico MTSK fundamenta la respuesta a dicha pregunta de investigación, teniendo en cuenta las evidencias del conocimiento de los EPP en sus respuestas al problema planteado, los indicios de dicho conocimiento (Escudero-Ávila, 2015) y las oportunidades (LiñánGarcía, 2017), entendiendo estas últimas como un elemento metodológico que nos va a permitir, como investigadores, identificar otros elementos del conocimiento especializado conectado con el evidenciado y que no aparece de manera explícita en estas respuestas, considerando nuestra sensibilidad teórica (Strauss y Corbin, 1994), que llamaremos en adelante conocimiento evocado.

El trabajo se enmarca en el paradigma interpretativo (Bassey, 1995), y en el contexto general de un estudio de caso de tipo instrumental (Stake, 2005), ya que permitirá una mejor comprensión del conocimiento para la enseñanza y el aprendizaje de la proporcionalidad compuesta, ayudando a la caracterización de este conocimiento para la formación de profesores. 
El diseño de investigación se caracteriza por: el planteamiento de problemas de proporcionalidad compuesta a EPP para que los resuelvan de manera justificada después de unas sesiones de clase en las que se han desarrollado contenidos relacionados con los mismos (Barrera-Castarnado, Liñán-García, Pérez-Bueno, 2017), la selección de respuestas de estudiantes con perfiles académicos equivalentes y el enfoque cualitativo con el que se abordan los procesos de recogida y análisis de los datos.

La recogida de la información se realiza a través de pruebas de evaluación escrita dentro de la asignatura Matemáticas específicas para maestros del primer curso del Grado en Educación Primaria del Centro de Estudios Superiores Cardenal Spínola CEU (centro adscrito a la Universidad de Sevilla), en las que se plantea la resolución de diferentes problemas de proporcionalidad compuesta. Se tendrá en cuenta tanto la respuesta a la pregunta del problema como las justificaciones que vayan haciendo durante el proceso de resolución, sean ambas correctas o no.

El problema seleccionado ha sido:

Cinco camiones, haciendo 6 viajes al día, consiguen evacuar $600 \mathrm{~m} 3$ de tierra en 4 días. ¿Cuántos días tardarán 7 camiones en mover $3500 \mathrm{~m} 3$ de tierra si desescombran en un vertedero más próximo, lo que permite a cada camión realizar 10 viajes al día?

Es un problema de proporcionalidad compuesta que involucra cuatro magnitudes (número de camiones por día, número de viajes por camión y por día, volumen y tiempo), siendo la medida de una de ellas dato o incógnita final dependiente de las otras tres: el tiempo, 4 días (dato), que tardan 5 camiones haciendo 6 viajes al día para descargar $600 \mathrm{~m} 3$ vs el tiempo (incógnita) que tardan 7 camiones haciendo 10 viajes al día para descargar $3500 \mathrm{~m} 3$. No hace falta señalar la obvia relación de dependencia (directa o inversa) que se establece entre todas ellas entre sí; serán los resolutores (EPP) quienes elijan, en cada caso, cuál de dichas relaciones van a utilizar para llegar a la solución pedida.

Podemos observar, además, que en el enunciado aparece una magnitud intensiva (el número de viajes por día). Por nuestra experiencia como formadores de EPP, apoyada además en investigaciones como las de Martínez et. al (2015), sabemos que el uso de dicho tipo de magnitudes, máxime cuando se involucran en proporcionalidades compuestas, suele conllevar mayores dificultades de interpretación. Se une a esto el hecho constatado por Martínez et al. (2017) de que la presencia de este tipo de problemas en los libros de texto de secundaria es prácticamente anecdótica, lo que podría tener como consecuencia la merma en su conocimiento en matemática fundamental, en el sentido de Ma (1999), con el que los EPP deberían llegar a la universidad. Si bien este contenido en sí mismo no forma parte de lo que los EPP deberán enseñar en Primaria, la resolución de este tipo de problemas forma parte del currículo del Grado en Educación Primaria de la Universidad de Sevilla porque, por un lado, el conocimiento del EPP ha de ser superior al que va a trabajar posteriormente con sus alumnos y, por otro, el conocimiento asociado a los problemas de proporcionalidad compuesta moviliza a su vez todo el conocimiento útil para la resolución de situaciones de proporcionalidad propias de Educación Primaria.

Como consecuencia de todo ello, consideramos que la propuesta de este problema a los EPP pondrá de manifiesto su conocimiento no solo acerca de la propia proporcionalidad, sino de las conexiones del mismo con otros campos dentro de la matemática, sin que se vea influido por la práctica sistemática.

Los informantes de este trabajo son EPP con resultados académicos equivalentes, particularmente en las pruebas de evaluación de la asignatura antes mencionada. Se han elegido entre los que han llegado a la solución correcta del problema un representante de cada tipo de estrategia 
Como ya hemos indicado, para el análisis del conocimiento especializado presente en las respuestas dadas por los EPP a las cuestiones planteadas nos basamos tanto en la identificación de evidencias e indicios (Escudero-Ávila, 2015) de elementos del MTSK, que como investigadores, interpretamos que se han movilizado, así como el que las respuestas de los estudiantes nos evoca (figura 2).

\section{Figura 2}

\section{MTSK en Proporcionalidad en la evaluación de pruebas para EPP}

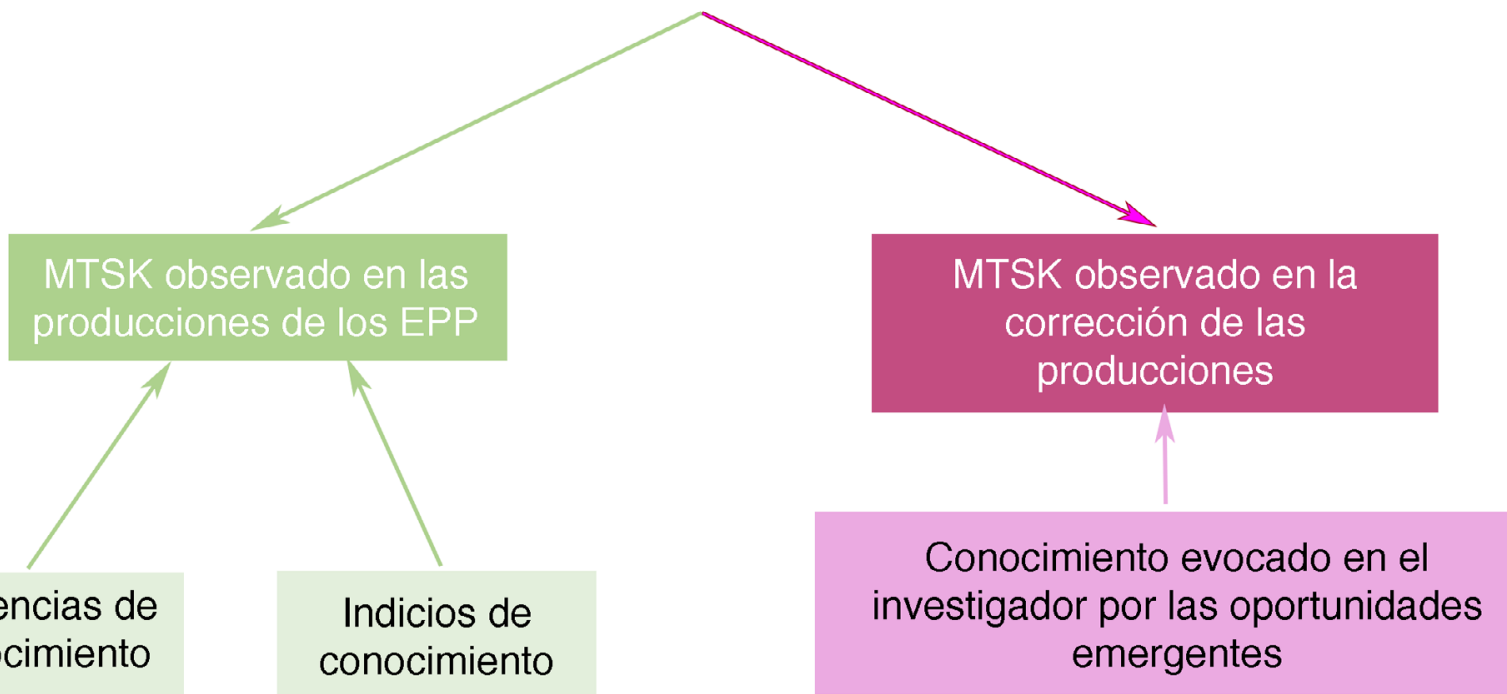

\section{ANÁLISIS DE LA INFORMACIÓN}

Para el análisis que presentamos hacemos uso de MTSK como modelo analítico para elaborar una descripción del conocimiento especializado que se moviliza en las respuestas de algunos EPP en la resolución de un problema de proporcionalidad compuesta. Debemos tener claro, como ya hemos manifestado en la metodología de este trabajo, que tal conocimiento vendrá dado tanto por las producciones de los EPP en sus pruebas de evaluación como por las correcciones de las mismas, donde podrá aparecer el conocimiento evocado al investigador (desde su sensibilidad teórica, Strauss y Corbin, 1994) por las oportunidades que emergen en dicha corrección. 


\section{Figura 3}

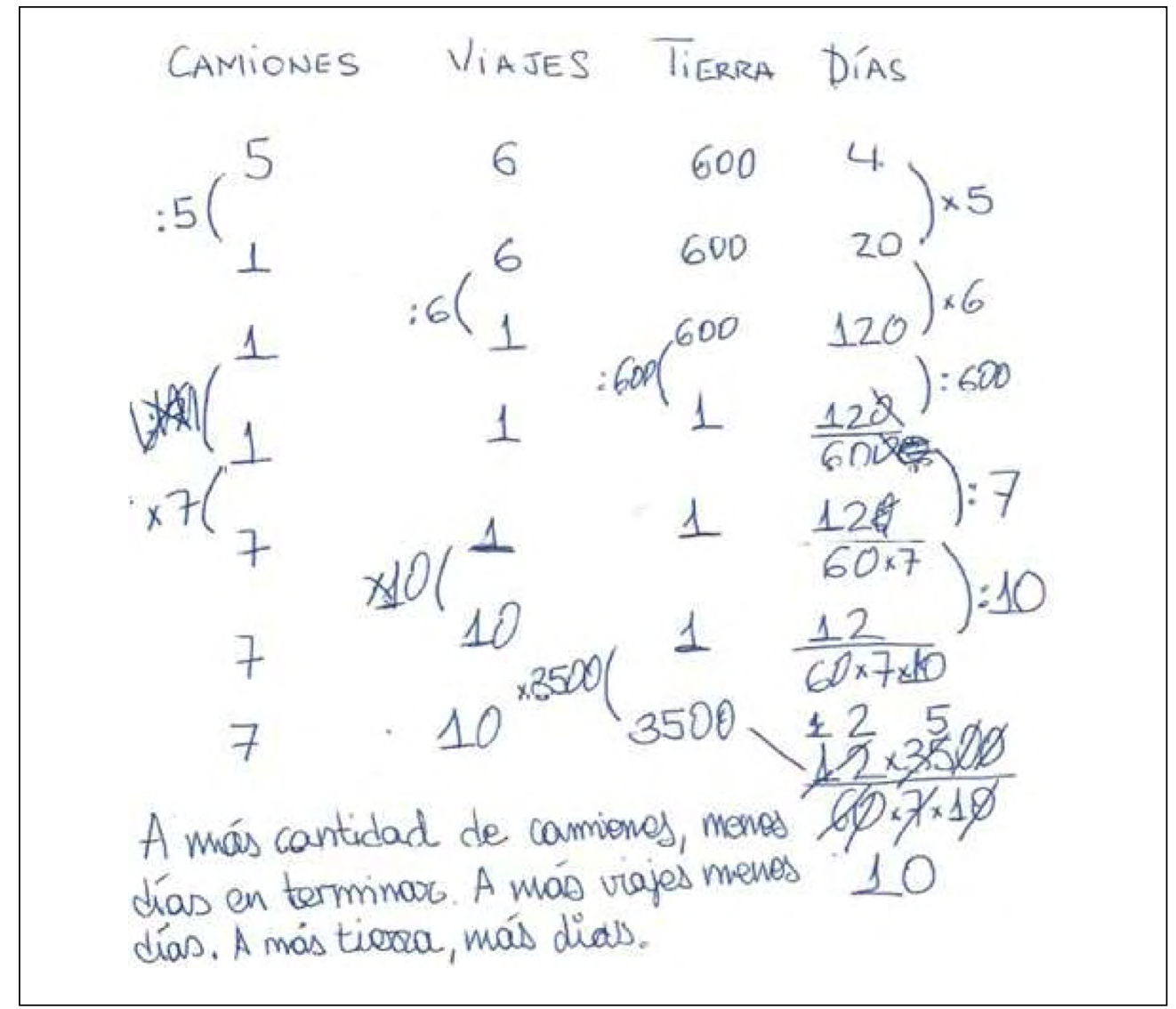

El estudiante 1 muestra una resolución basada en el método de reducción a la unidad, en el que elige una a una cada magnitud independiente -mientras que el resto de estas quedan constantes o invariables- y va realizando divisiones que le permitirán llegar a la medida de una unidad para cada una de ellas, lo que implicará, a su vez, una transformación de la magnitud dependiente con una multiplicación (relación inversa) o una división (relación directa). Por ejemplo, la primera modificación que propone deja invariantes el número de viajes por día y el volumen de tierra evacuado, mientras divide para obtener el valor del número de camiones y multiplica el tiempo por el mismo valor por el que dividió tal primera magnitud (ver primera y segunda fila de la figura 3). De esta forma consigue la medida de la magnitud dependiente tiempo para la medida una unidad en todas las magnitudes independientes, o lo que es lo mismo, habiendo reducido a la unidad la medida de cada una de estas últimas.

Una vez conseguido, multiplica cada una de las medidas de las magnitudes independientes por el número que corresponde a las medidas de las mismas que propone el problema. Así, la medida de la magnitud dependiente vuelve a ser transformada con una multiplicación (relación inversa) o una división (relación directa), llegando a la solución correcta del problema. Siguiendo con el ejemplo anterior, en esta ocasión mantiene las mismas invariantes, mientras que multiplica para obtener el valor 7 camiones en la primera magnitud y divide el tiempo por ese mismo valor (ver cuarta y quinta fila de la figura 3). 


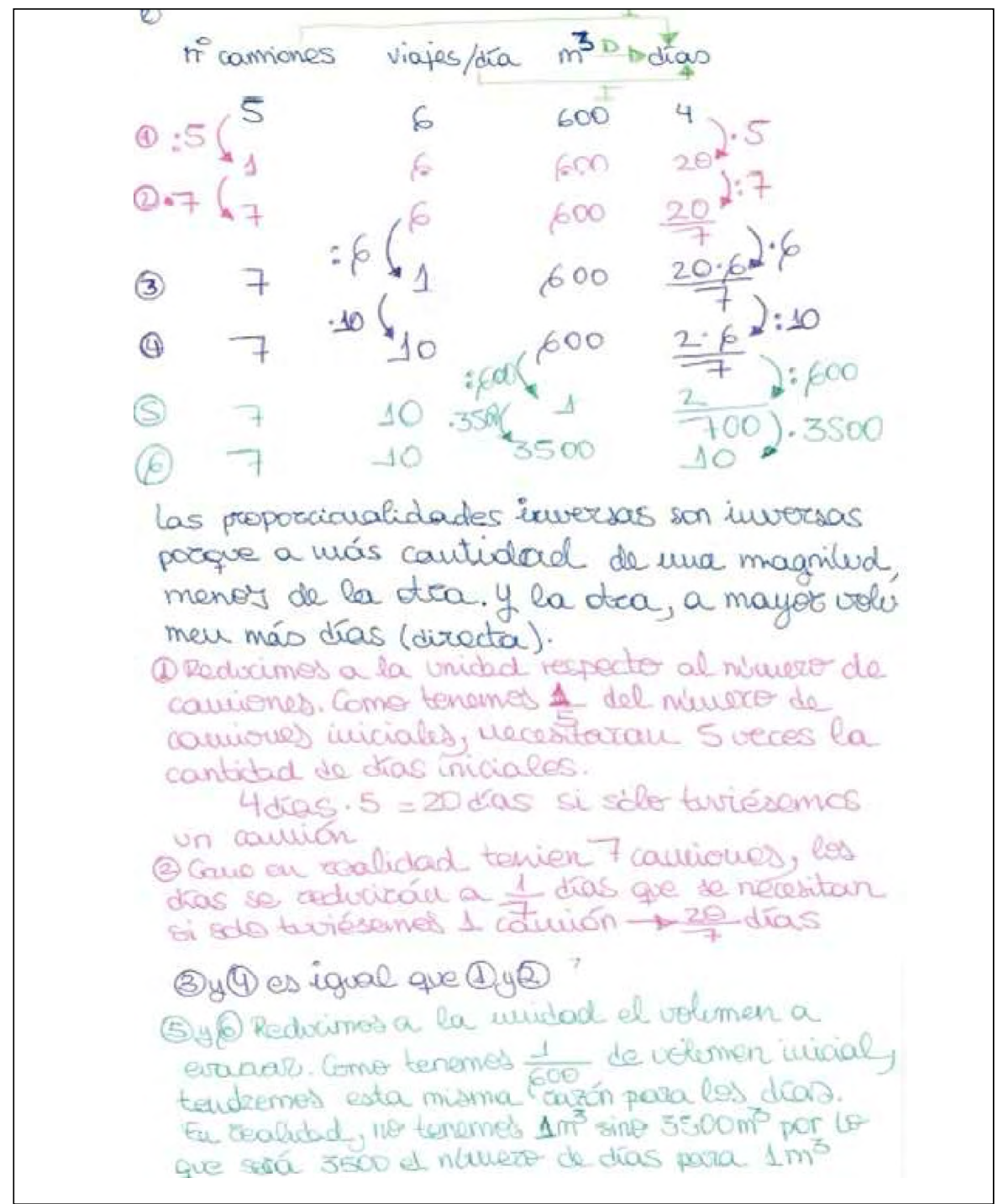

Se evidencia un KoT procedimientos, en el que aparece el cómo se hace, que incluye tanto reducción a la unidad como las operaciones que se involucran en tal reducción. Muestra, además en este indicador, el conocimiento de la necesaria jerarquización del procedimiento, pero debemos analizar la justificación de la resolución para comprobar si los indicios sobre su conocimiento de ¿cuándo puede hacerse?, ¿por qué se hace así? y características del resultado se manifiestan como evidencias de esta categoría. También observamos un KoT registros de representación simbólica en este caso (conocimiento observado también en el resto de los estudiantes analizados). Emerge una oportunidad que evoca la conexión de complejización (KSM) entre la definición de operación (en este caso, producto) desde la conceptualización como suma reiterada hasta como razón.

El estudiante se limita a describir el procedimiento llevado a cabo sin una justificación sobre el mismo. Es decir, no explicita por qué puede llevarse a cabo, pues solo indica que una división en una medida de la primera magni- 
tud independiente implica una multiplicación en la dependiente porque a más cantidad de camiones, menos días en terminar, no estableciendo, por tanto, la relación proporcional entre las mismas (ver justificación en figura3). Si en las indicaciones de las operaciones realizadas se hubiera mostrado, por ejemplo, la necesidad de la ausencia de unidades de las cantidades por las que se multiplica o divide (cuantificadores) una medida de alguna de las magnitudes, se habría podido evidenciar un KoT procedimientos características de los resultados parciales. Asimismo, el cuándo y por qué se hace así de esta categoría, se podrían haber mostrado al hacer patente la relación proporcional y al conectar la relación multiplicativa entre las medidas de las magnitudes con la relación proporcional. Si el estudiante hubiera explicitado el significado de las no unidades de medida en un cuantificador, nos habría dado información sobre su conocimiento acerca de la definición, propiedades y sus fundamentos (KoT) del mismo como razón escalar (KoT fenomenología). Además, observamos que en la cabecera de la tabla (figura 3) no aparecen ni magnitudes ni unidades en sentido estricto, sino una designación de elaboración propia partiendo del enunciado del problema que le ayuda a organizar los datos; emerge, entonces, el conocimiento evocado correspondiente al registro de representación (KoT), que está a su vez relacionado con definiciones, propiedades y sus fundamentos (KoT) de las magnitudes y las unidades de medida asociadas.

El estudiante 2 muestra un procedimiento equivalente al del estudiante 1, si bien en lugar de elegir una a una cada una de las magnitudes independientes -mientras que el resto de las magnitudes independientes quedan constantes o invariables- e ir realizando las divisiones que le permitirán llegar a la medida de una unidad para cada una de ellas, elige una magnitud que reduce a la unidad y, seguidamente, multiplica por el cuantificador que le permitirá conseguir la cantidad que propone el problema. Modifica, por tanto, en dos veces sucesivas la medida de la magnitud dependiente. Por ejemplo, la primera modificación que propone deja invariantes el número de viajes por día y el volumen de tierra evacuado, mientras divide para obtener el valor 1 camión en la primera magnitud y multiplica el tiempo por el mismo valor por el que dividió tal primera magnitud. A continuación, dejando las mismas invariantes, multiplica para obtener el valor 7 camiones en la primera magnitud, dividiendo el tiempo consecuentemente por ese mismo valor (ver primera, segunda y tercera fila de la figura 4).

Podemos ver que se muestran las mismas evidencias que en el primer estudiante, si bien el número de las mismas aumenta en este segundo estudiante pues algunos indicios se tornan evidencias en la justificación que propone. Por ejemplo, al decir como tenemos 1/5 del número de camiones iniciales, necesitarán 5 veces la cantidad de días iniciales. Pero como, en realidad, tenemos 7 camiones, los días se reducirán a 1/7 de los días que se necesitarían si solo tuviésemos un camión. A pesar de que no justifica previamente que sean magnitudes proporcionales más allá de lo que hace el estudiante 1 (por tanto sigue sin haber evidencias del cuándo puede hacerse), sí queda constancia de su conocimiento sobre el por qué puede hacerse y las características, si bien parciales, del resultado (KoT procedimientos). En el ejemplo anterior también hay indicios de conocimiento del significado de cuantificador (KoT definiciones, propiedades y sus fundamentos) al indicar el significado del producto del cuantificador como un número de veces la medida de la magnitud.

Emerge en este caso el conocimiento evocado que permite fundamentar las relaciones de proporcionalidad entre magnitudes atendiendo a las que se establecen entre sus unidades de medida (KSM simplificación), en el hecho de reconocer que para pasar de tener 5 camiones a tener 1 camión hay que dividir por un cuantificador que mantendrá las mismas unidades de medida. 
Figura 5

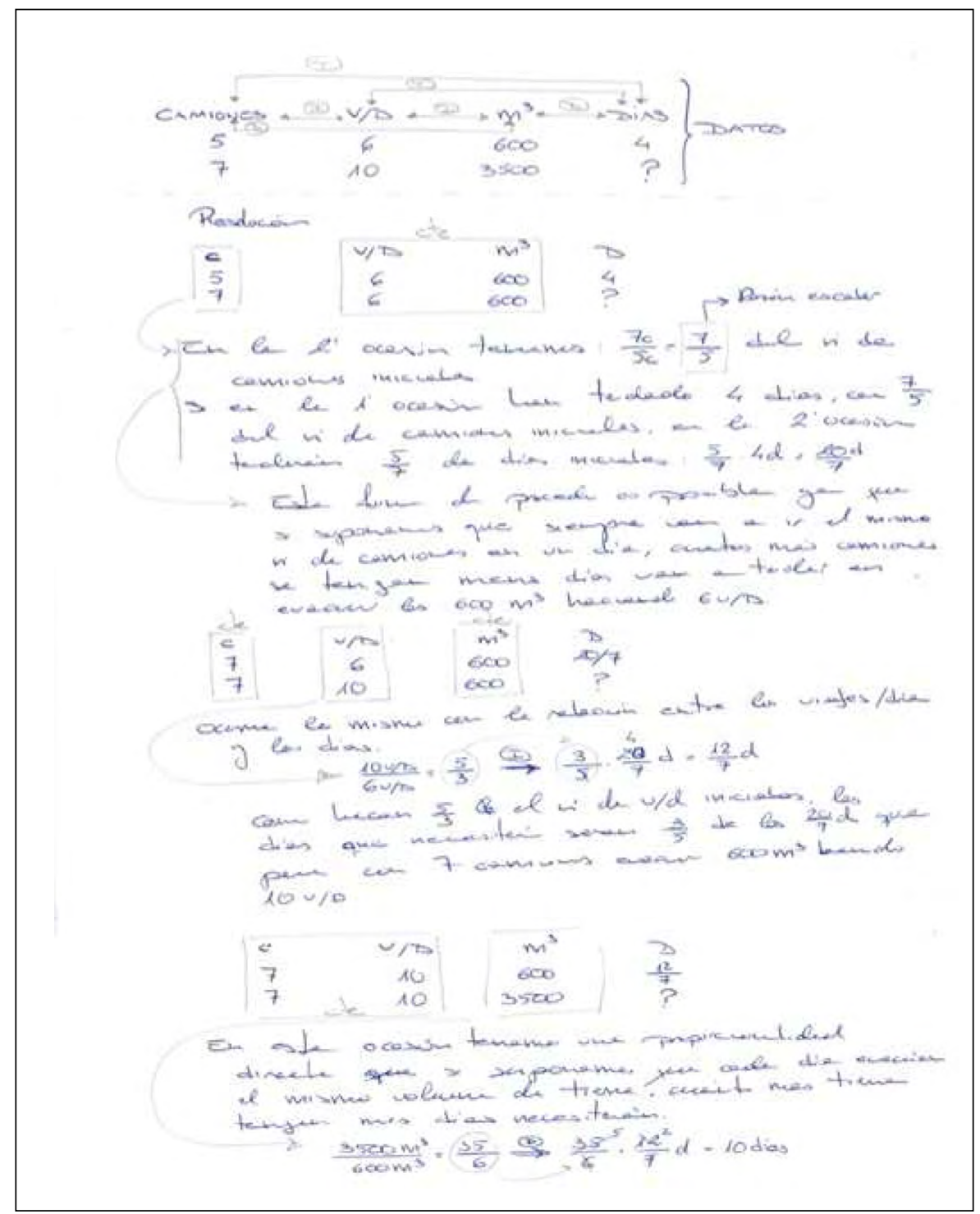

El estudiante número 3 utiliza la razón escalar como herramienta para la resolución del problema; es decir, compara las medidas de las mismas magnitudes para obtener las veces de una cantidad frente a la otra. Por ejemplo, inicialmente hay 5 camiones que, posteriormente, suben a 7 camiones; el estudiante representa la relación diciendo que en la segunda ocasión hay 7/5 del número de camiones iniciales. Una vez hecho esto, y tras justificar la relación (proporcionalidad inversa) entre las magnitudes número de camiones y tiempo, multiplica por la inversa de la fracción indicada, razonando que si en la primera ocasión han tardado cuatro días, en la segunda ocasión, con $7 / 5$ del número de camiones iniciales, tardarán $5 / 7$ de los días iniciales (ver figura 5 , justificación). Repite el proceso de relación con la magnitud dependiente para el resto de las magnitudes independientes; en lugar de repetir la explicación, identifica la primera con las necesarias para el resto de los pasos. 
Este estudiante muestra, razonadamente, la relación que existe entre cada magnitud independiente con la dependiente, así como el resto de relaciones (aunque estas últimas no las utiliza). Evidencia un KoT procedimientos, en este caso mostrando conocimiento sobre el ¿cómo se hace?, puesto que utiliza un procedimiento adecuado y llega satisfactoriamente al resultado; ¿cuándo puede hacerse? (lo que en los estudiantes 1 y 2 no aparecía) y ¿por qué se hace así? (que ya aparecía en el estudiante 2), ya que hace uso de la razón escalar una vez justificado el significado de la misma; y características del resultado, analizando en cada paso el sentido de sus conclusiones parciales. En este caso, a diferencia de los anteriores, sí se evidencia KoT definiciones, propiedades y sus fundamentos al indicar el significado de la relación escalar entre medidas de una magnitud, usando la misma unidad en cada una de ellas, y convirtiendo, de esta forma, la razón en un cuantificador adimensional (figura 5, primera justificación).

Observamos un indicio de conocimiento de la conexión de complejización (KSM) existente entre la razón escalar y la medida, cuando compara el número de camiones entre la segunda ocasión y la primera, indicando que una es $7 / 5$ respecto a la otra.

En la respuesta de los estudiantes 2 y 3 emerge el conocimiento evocado sobre la conexión entre la relación multiplicativa entre dos cantidades y su uso como modelo atribuible al cálculo de la razón de proporcionalidad (KoT fenomenología).

\section{Figura 6}

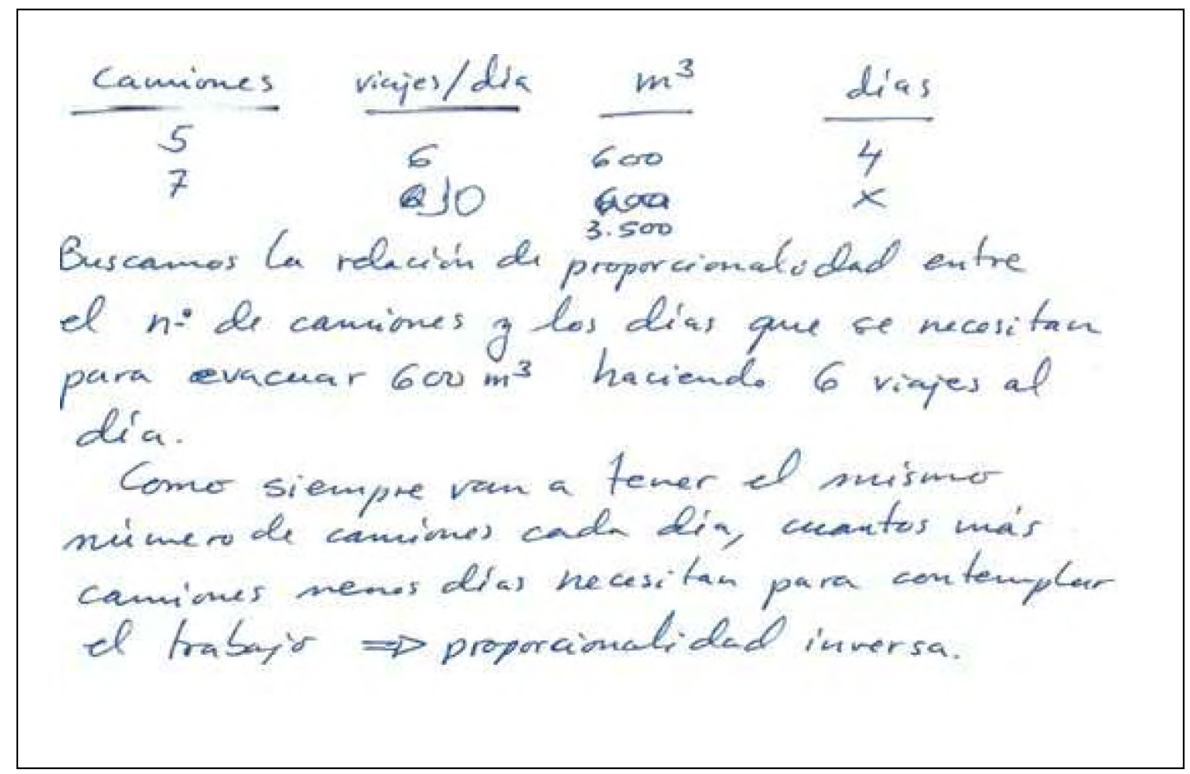

El estudiante 4, al igual que el anterior, hace una justificación adecuada sobre las relaciones entre las magnitudes, pero solo las que va a utilizar, es decir, las existentes entre cada una de las independientes y la dependiente. Hace uso de la razón funcional entre cada una de las magnitudes independientes con la dependiente, buscando así las constantes de proporcionalidad entre dichas magnitudes. Al contrario que otros estudiantes, este relaciona medidas de magnitudes en una misma situación entre sí. Por ejemplo, 5 camiones iniciales viajando 4 días se convierte 
en 5camiones 4 días=20camiones día para la relación inversa entre estas magnitudes. Una vez encontradas las razones de proporcionalidad las utiliza para relacionar distintas situaciones. Por ejemplo, como en la segunda situación tenemos 7 camiones, decide buscar cuántos grupos de 7 camiones se pueden formar con los 20 camiones que viajarían si solo tuvieran un día disponible. Consigue la constante de proporcionalidad, en el caso de la relación directa entre las magnitudes volumen y tiempo, dividiendo las medidas de ambas magnitudes (figura 6).

Todo el conocimiento evidenciado en la producción de los estudiantes anteriores queda patente también en este caso; sin embargo, es el primero que evidencia conocimiento sobre las unidades de medida de las magnitudes involucradas (KoT definiciones, propiedades y sus fundamentos), por ejemplo, al hacer explícita la siguiente afirmación: la constante de proporcionalidad funcional entre estas dos magnitudes nos dará el número de camiones si solo tuviéramos un día. Para calcularlo multiplicamos 5camiones-4días=20 camiones·día. Explica esta relación haciendo un gráfico (KoT registro de representación) en el que se puede observar la unidad día dividida en cuatro partes, cada una de las cuales representa cinco camiones (ver figura 7), dejando patente un indicio sobre la conexión transversal (KSM) entre la proporcionalidad inversa y la interpretación de la unidad en los números racionales.

\section{Figura 7}

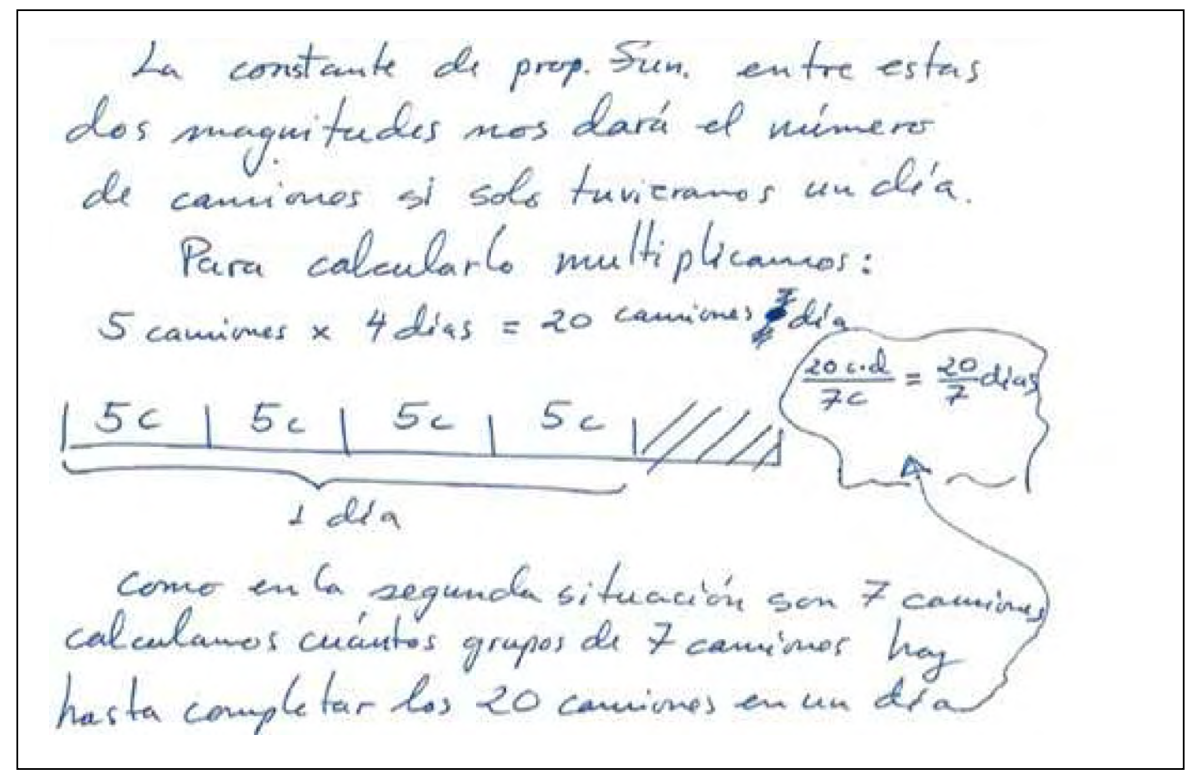

Además, observamos otro indicio, esta vez una conexión auxiliar (KSM) al hacer uso de la división medida -20camiones·día/7camiones- o división reparto $-600 \mathrm{~m}^{3} /((2 \cdot 6 / 7)$ días $)$ - como herramienta para el cálculo de las constantes de proporcionalidad.

Destacamos que en todos los casos los estudiantes han mantenido los resultados parciales en expresiones fraccionarias, intentando realizar simplificaciones (figura 8) siempre que ha sido posible (KoT registros de representación). 
Figura 8

Producción estudiante 4 (cálculo con simplificación)

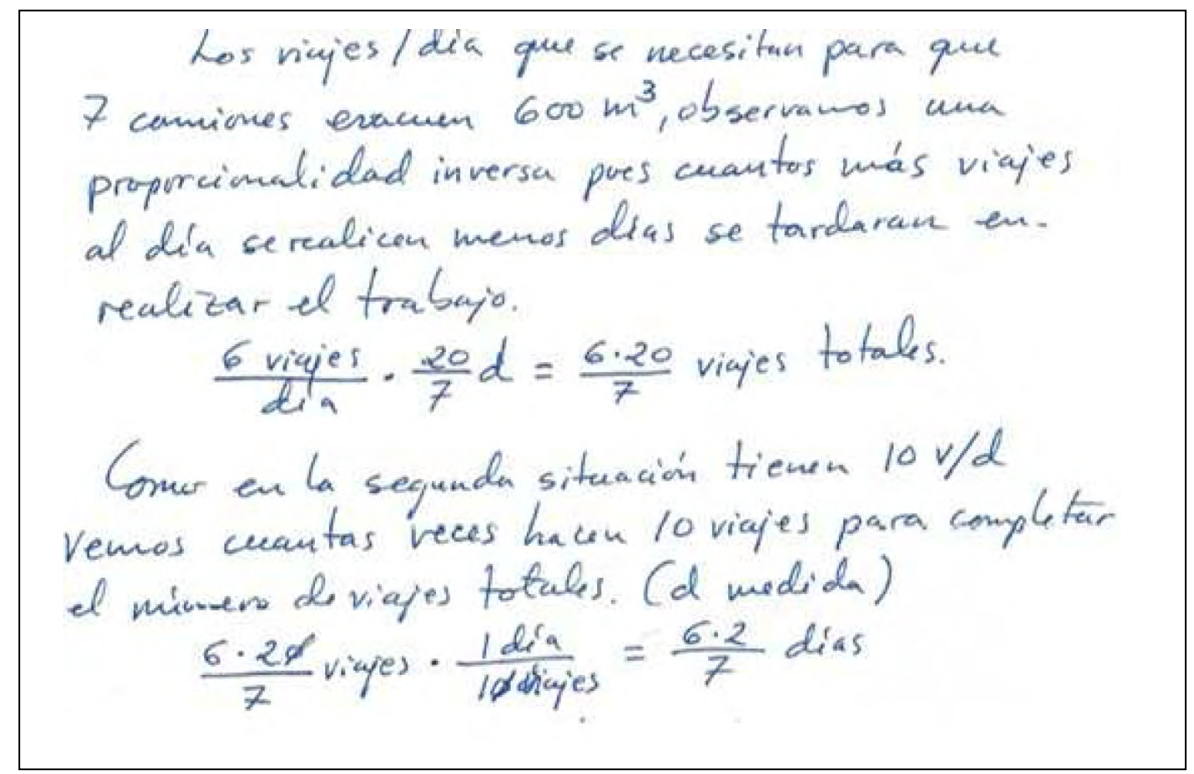

Asimismo, por un lado se ha evidenciado un KPM papel de los simbolos y uso del lenguaje formal en cuanto a que utilizan adecuadamente el lenguaje algebraico, y por otro, existen indicios en el mismo subdominio sobre el indicador jerarquización y planificación como forma de proceder en la resolución de problemas matemáticos, pues van mostrando su plan de ejecución de manera ordenada (figura 9).

Figura 9

Para la relacion volumen-dias come san a evacual el mismo volumen de tierra cada día. seanta wás tierra unis dias necesstare wor $\Rightarrow$ prep. direta

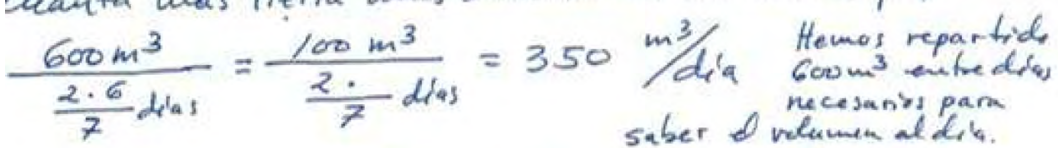

Tenemor que medir céantas veces entran $550 \mathrm{~m}^{3}$ en $3.500 \mathrm{~m}^{3}$ ya que cada vez que exruen $350 \mathrm{~m}^{3}$ pasará un d'a

3. $500 \mathrm{~m}^{3} \cdot \frac{1 d / a}{350 \mathrm{~m}^{3}}=10$ dias

Se necesitan 10 dies para que 7 camioner evawen $3.500 \mathrm{~m}^{3}$ hacien do $10 \mathrm{viajes} / \mathrm{dia}$. 


\section{CONCLUSIONES}

A pesar de que todos los estudiantes consiguen llegar a la solución correcta, observamos que sus estrategias y justificaciones son muy diferentes. Mientras que el primero solo deja evidencias claras sobre su KoT procedimientos en cuanto al indicador ¿cómo se hace?, progresivamente se observa cómo los demás van evidenciando su conocimiento sobre el cuándo puede hacerse, por qué se hace así y características del resultado. Es el cuarto quien evidencia su conocimiento sobre todos los indicadores de esta categoría, con la particularidad, además de que evidencia conocimiento sobre las características del resultado tanto en los resultados parciales como en el final.

En todos los casos los estudiantes han mantenido los resultados parciales en expresiones racionales, intentando realizar simplificaciones siempre que ha sido posible (KoT registros de representación) en distintos niveles de ejecución (es de destacar la producción del segundo estudiante al respecto, ver figura 5). Parece que son conscientes de la ventaja que supone este registro de representación frente a la realización indiscriminada de operaciones.

Tal y como muestran Martínez et al. (2015), les resulta complicado dar significado a las razones de proporcionalidad inversa que generan unidades del tipo camiones día, que indican la cantidad de camiones que serían necesarios si solo hubiese un día para poder trabajar o la cantidad de días que serían necesarios si solo hubiese un camión disponible.

Ninguno de los estudiantes que realizaron la prueba usó el producto de magnitudes para reducir el número de las mismas, lo que no nos ha dejado evidencias del conocimiento que se podría movilizar en este caso.

Se puede ver una variación gradual sobre los indicios que tenemos del indicador prácticas particulares del quehacer matemático (KPM) desde el estudiante 1, que se limita a realizar una descripción del procedimiento, al estudiante 4 que muestra la importancia de la justificación para validar el resultado (KPM procesos asociados a la resolución de un problema como forma de producir matemáticas). Esta misma situación se refleja en la designación (KoT registros de representación) que cada uno de ellos realiza de las magnitudes, lo que evidencia sus debilidades y fortalezas en la concepción de las mismas (KoT definiciones, propiedades y sus fundamentos): mientras el estudiante 1 designa cada columna de datos con los términos que le sugiere el enunciado, alejados de la identificación de la magnitud, y no hace referencia a las unidades de medida de ninguna de ellas en fase alguna de la resolución, el estudiante 4 designa apropiadamente cada una de las magnitudes y les asocia unidades apropiadas. Los estudiantes 2 y 3 están en un punto intermedio entre ambos.

Dando respuesta a nuestra pregunta de investigación, ¿qué conocimiento especializado puede identificarse en el análisis de producciones de EPP cuando resuelven problemas de proporcionalidad numérica compuesta? vemos que cuanto mayor uso hacen de las unidades de medida para dar significado a las magnitudes y a las relaciones existentes entre ellas, las justificaciones que acompañan al procedimiento son más exhaustivas, mostrando, entonces, una mayor riqueza en el conocimiento especializado evidenciado en la proporcionalidad entre magnitudes.

A partir de aquí, como prospectiva de esta investigación, nos planteamos el diseño de actividades que involucren problemas de proporcionalidad en los que se potencie el conocimiento relacionado con las magnitudes y las unidades de medida que se pueden asociar a ellas teniendo en cuenta tanto los indicios de conocimiento especializado que han surgido como el conocimiento evidenciado y evocado, teniendo como fin último la caracterización del conocimiento especializado en proporcionalidad para los profesores de Educación Primaria. 


\section{REFERENCIAS}

Aguilar, A. (2015). El conocimiento especializado de una maestra sobre la clasificación de las figuras planas. Un estudio de caso. Tesis doctoral:Universidad de Huelva. Recuperado de: http://rabida.uhu.es/dspace/handle/10272/12006.

Ball, D. L., Thames, M. H., y Phelps, G. (2008). Content knowledge for teaching: What makes it special? Journal of Teacher Education, 59(5), 389-407.

Barrera-Castarnado, V. J., Liñán-Gacía, M. M. y Pérez-Bueno, B. (2017). El conocimiento especializado de los estudiantes para maestro en la resolución de problemas de magnitudes proporcionales: una propuesta didáctica. En J. Carrillo, L. C. Contreras y M. Montes (Eds.), Actas de las III Jornadas del Seminario de Investigación de Didáctica de la Matemática de la Universidad de Huelva. SGSE: Huelva.

Bassey, M. (1995). Creating Education Through Research. Edimburgo: British Educational Research Association.

Buforn, A., Fernández, C. y Llinares, S. (2015). El papel del conocimiento de matemáticas en la identificación de la comprensión de los estudiantes: el significado de razón. Comunicación presentada en el XIV CIAEM, Tuxtla Gutiérrez, Chiapas, México. Mayo.

Buforn, A. y Fernández, C. (2014). Conocimiento de matemáticas especializado de los estudiantes para maestro de primaria en relación al razonamiento proporcional. Boletim de Educaçao Matemática (BOLEMA), 28(48), 21-41.

Carrillo, J., Climent, N., Montes, M., Contreras, L .C., Flores-Medrano, E., Escudero-Ávila, D., Vasco, D., Rojas, N., Flores, P., Aguilar-González, Á., Ribeiro, M. y Muñoz-Catalán, M. C. (2018). The Mathematics Teacher's Specialised Knowledge (MTSK) model. Research in Mathematics Education. https://doi.org/10.1080/14794802 .2018.1479981.

Escudero-Ávila, D. I. (2015). Una caracterización del conocimiento didáctico del contenido como parte del conocimiento especializado del profesor de matemáticas de secundaria. Tesis doctoral: Universidad de Huelva. Recuperado de http://rabida.uhu.es/dspace/handle/10272/11456.

Fernández, C. y Llinares, S. (2011). De la estructura aditiva a la multiplicativa: efecto de dos variables en el desarrollo del razonamiento proporcional. Infancia y Aprendizaje, 34(1) 67-80.

Flores-Medrano, E. (2015). Una profundización en la conceptualización de elementos del modelo de Conocimiento Especializado del Profesor de Matemáticas (MTSK). Tesis doctoral: Universidad de Huelva. Recuperado de http://rabida.uhu.es/dspace/handle/10272/11503.

González, M. J. y Gómez, P. (2011). Magnitudes y medida. Medidas directas. En I. Segovia y L. Rico (Coords.), Matemáticas para maestros (pp. 351-374). Madrid, España: Pirámide.

Lesh, R. (1997). Matematización: la necesidad "real" de la fluidez en las representaciones. Enseñanza de las Ciencias, 15(3), 377-391.

Liñán-García, M. M. (2017). Conocimiento Especializado en Geometría en un aula de $5^{\circ}$ de Primaria. Tesis doctoral: Universidad de Huelva. Recuperado de http://rabida.uhu.es/bitstream/handle/10272/14230/ Conocimiento_especializado.pdf? sequence $=6$

Ma, L. (1999). Knowing and teaching elementary mathematics: teachers' understanding of fundamental mathematics in China and the United States. Mahwah. NJ: Lawence Erlbaum.

Martínez, S., Muñoz, J. M. y Oller, A. M. (2015). Estrategias utilizadas por estudiantes de distintos niveles educativos ante problemas de proporcionalidad compuesta. En C. Fernández, M. Molina y N. Planas (eds.), Investigación en Educación Matemática XIX (pp. 351-359). Alicante: SEIEM.

Martínez, S., Muñoz, J. M., Oller, A. M. y Ortega, T. (2017). Análisis de problemas de proporcionalidad compuesta en libros de texto de $2^{\circ}$ de ESO. Revista Latinoamericana de Investigación en Matemática Educativa (RELIME), 20(1), 95-122. 
Montes, M. (2015). Conocimiento Especializado del Profesor de Matemáticas acerca del infinito. Un estudio de caso. Tesis doctoral: Universidad de Huelva. Recuperadode http://rabida.uhu.es/dspace/bitstream/handle/10272/10944/ Conocimiento_especializado.pdf? sequence $=4$

Montes, M., Aguilar, A., Carrillo, J. y Muñoz-Catalán, M. C. (2014). MTSK: From common and horizon knowledge to knowledge of topics and structures. En B. Ubuz, C. Haser y M. A. Mariotti (Eds.), Actas del Cerme 8 (pp. 3185-3194). Antalya, Turquía: ERME.

Moriel-Junior, J. G. (2014). Conhecimento especializado para ensinar divisão de frações. Tesis doctoral: Universidad de Huelva. Recuperado de: http://www.ufmt.br/ufmt/unidade/userfiles/ publicacoes/79e3fe1d66c40ff5d174dc92c84fc777.pdf

Muñoz-Catalán, M. C., Liñán-García, M. M., Ribeiro, C. M. (2017). Conocimiento especializado para enseñar la operación de resta en Educación Infantil. Cadernos de Pesquisa, 24, n. Especial, set./dez. 2017. http:// dx.doi.org/10.18764/2178-2229.v24nespecialp4-19

NCTM (2000). Principles and Standards for School Mathematics. Traducción SAEM Thales (2003). Principios y Estándares para la Educación Matemática. Sevilla: SAEM Thales.

Ribeiro, C. M., Muñoz-Catalán, M. C., y Liñán-García, M. M. (2015). Discutiendo el conocimiento matemático especializado del profesor de infantil como génesis de aprendizajes futuros. En I. M. Gómez-Chacón, J. Escribano, A. Kuzniak y P. R. Richard. Actas cuarto congreso internacional ETM (pp. 575-590), Universidad Complutense de Madrid, San Lorenzo del Escorial: ETM4. Recuperado de http://www.mat.ucm.es/imi/ ETM4/ETM4libro-final.pdf

Rivas, M. A. Godino, J. D. y Castro, W. F. (2012). Desarrollo del conocimiento para la enseñanza de la proporcionalidad en futuros profesores de primaria. Boletim de Educaçao Matemática (BOLEMA), 26(42 B), 559-588.

Rojas, N. (2014). Caracterización del conocimiento especializado del profesor de matemáticas: un estudio de casos. Tesis doctoral: Universidad de Granada. España.

Sánchez, E. A. (2013). Razones, proporciones y proporcionalidad en una situación de reparto: Una mirada desde la teoría antropológica de lo didáctico. Revista Latinoamericana de Investigación en Matemática Educativa, 16(1), 65-97.

Schoenfeld, A. H., y Kilpatrick, J. (2008). Toward a theory of proficiency in teaching mathematics. En D. Tirosh y T. Woods (Eds.), Tools and processes in Mathematics Teacher Education. International handbook of mathematics teacher education 2 (pp. 321-354). Roterdam: Sensepublisher.

Schoenfeld, A. H. (2010). How we think. Nueva York: Routledge.

Shulman, L. S. (1986). Those who understand: knowledge growth in teaching. Educational Researcher, 15(2), 4-14.

Stake, R. E. (2005). Qualitative Case Studies. En N. K. Denzin y Y. S. Lincoln (Eds.), The Sage handbook of qualitative research. Third edition (pp. 443-166). Thousand Oaks: Sage Publications

Star, J. y Rittle-Johnson, B. (2009). Making algebra work: Instructional strategies that deepen student understanding, within and between representations. ERS Spectrum, 27 (2), 11-18.

Strauss, A., y Corbin, J. (1994). Grounded Theory Methodology. En N. K. Denzin y Y. S. Lincoln (Eds.) Handbook of Qualitative Research (pp. 217-285). Thousand Oaks: Sage Publications.

Vasco, D. L. (2015). Conocimiento especializado del profesor de álgebra lineal: un estudio de casos en el nivel universitario. Tesis doctoral: Universidad de Huelva. Recuperado de http://rabida.uhu.es/dspace/handle/10272/11901

Valverde, A. G. y Castro, E. (2009). Actuaciones de maestros en formación en la resolución de problemas de 


\section{INFORMACIÓN SOBRE LOS AUTORES}

Beatriz Pérez Bueno. Licenciada en Ciencias Físicas por la Universidad de Zaragoza, Máster en Investigación en la Enseñanza y el Aprendizaje de las Ciencias Experimentales, Sociales y Matemáticas y realizando su tesis doctoral por la universidad de Huelva bajo la línea: Formación inicial y desarrollo profesional del profesorado en Didácticas Específicas. Sus intereses de investigación están orientados a la enseñanza y el aprendizaje de aquellos fenómenos y conceptos físicos en los que exista una relación de proporcionalidad entre sus magnitudes, utilizando las unidades de medida como herramienta significativa dejando, así, a un lado el uso memorístico de fórmulas algebraicas. Actualmente es docente en el Centro Cardenal Spínola CEU, tanto en el Grado de Educación Primaria, como de Educación Infantil.

$\triangle$ bperez@ceuandalucia.es

Ma Mar Liñán García. Doctora en Didáctica de las Matemáticas por la Universidad de Huelva, Profesora Titular del Área de Didáctica de la Matemática en el Centro Cardenal Spínola CEU (adscrito a Universidad de Sevilla) y Profesora Asociada de la Universidad de Sevilla en la misma área. Miembro del Study Group del Real Colegio Complutense at Harvard Studying and improving Mathematics instruction in secondary schools in Spain (SiMiS), de la Red temática de Excelencia RED8-Educación Matemática y Formación de profesores, del Grupo de investigación en Educación Matemática (GIEM) Universidad de Sevilla: FQM226, de la Red Iberoamericana MTSK y del Seminario de Investigación en Didáctica de la Matemática, SIDM, de la Universidad de Huelva, siendo su línea de investigación el conocimiento y desarrollo profesional del profesor de matemáticas.

\section{$\triangle$ mlinan@ceuandalucia.es}

Víctor Barrera Castarnado. Licenciado en Matemáticas por la Universidad de Granada. DEA presentado en el Departamento de Didáctica de la Matemática de la Universidad de Granada. Doctorando en el Programa IECAD de la Universidad de Huelva. Profesor del Área de Matemáticas del Centro de Estudios Superiores Cardenal Spínola- CEU desde octubre de 1995 y Profesor Asociado del Departamento de Didáctica de la Matemática de la Universidad de Sevilla desde 2010. Miembro del Study Group Studying and improving Mathematics instruction in secondary schools in Spain(SiMiS) del Real Colegio Complutense at Harvard. Miembro de la Red temática de Excelencia RED8-Educación Matemática y Formación de profesores. Miembro del Grupo de investigación en Educación Matemática (GIEM) Universidad de Sevilla: FQM226. Miembro de la Red Iberoamericana MTSK. Miembro del Seminario de Investigación en Didáctica de la Matemática, SIDM, de la Universidad de Huelva. Participa actualmente en el Proyecto de Innovación docente El desarrollo profesional del formador de maestros desde la elaboración y análisis de la puesta en práctica de casosde la Universidad de Huelva.

Líneas de investigación: "Conocimiento y Desarrollo Profesional del Profesor de Matemáticas".

\section{vbarrera@ceuandalucia.es}

